Research Article

\title{
The Impact of Preoperative Opioid Use Disorder on Complications and Costs following Primary Total Hip and Knee Arthroplasty
}

\author{
Jacob M. Wilson $\mathbb{D}^{1},{ }^{1}$ Kevin X. Farley, ${ }^{1}$ Matthew Aizpuru, ${ }^{2}$ Eric R. Wagner, ${ }^{1}$ \\ Thomas L. Bradbury, ${ }^{1}$ and George N. Guild ${ }^{1}$ \\ ${ }^{1}$ Department of Orthopaedic Surgery, Emory Orthopaedics \& Spine Center, 59 S Executive Park NW, Atlanta, GA 30329, USA \\ ${ }^{2}$ Emory School of Medicine, 201 Dowman Dr., Atlanta, GA 30322, USA \\ Correspondence should be addressed to Jacob M. Wilson; jacobmwilson12@gmail.com
}

Received 14 August 2019; Accepted 29 November 2019; Published 20 December 2019

Academic Editor: Panagiotis Korovessis

Copyright (c) 2019 Jacob M. Wilson et al. This is an open access article distributed under the Creative Commons Attribution License, which permits unrestricted use, distribution, and reproduction in any medium, provided the original work is properly cited.

\begin{abstract}
Introduction. Multiple studies have demonstrated that patients taking opioids in the preoperative period are at elevated risk for complications following total hip (THA) and knee (TKA) arthroplasty. However, the incidence and impact of opioid use disorder (OUD) among these patients-both clinically and fiscally-remain unknown. The purpose of this study was to investigate this relationship. Methods. The Nationwide Readmission Database (NRD) was used to identify patients undergoing THA and TKA from 2011 to 2015. Coarsened exact matching was used to statistically match the OUD and non-OUD cohorts. Further analysis was then conducted on matched cohorts with multivariate analysis. The incidence of OUD was also determined, and the costs associated with this comorbidity were calculated. Results. The incidence of OUD in arthroplasty patients increased $80 \%$ over the study period. OUD patients had higher odds of prosthetic joint infection (OR 1.55, 95\% CI 1.23-1.94), wound complication (OR 1.40, 95\% CI 1.12-1.76), prosthetic complication (OR 1.37, 95\% CI 1.10-1.70), and revision surgery (OR 1.47, 95\% CI 1.19-1.81). OUD patients also had longer length of stays (TKA: +0.67 days; THA: +1.09 days), higher readmission (OR 1.60, 95\% CI 1.43-1.79), and increased 90-day costs (TKA: $+\$ 3,602$ [95\% CI \$3,138-4,065]; THA: $+4,527$ [95\% CI \$3,593-4,920). Conclusion. Opioid use disorder is becoming a more common comorbidity among THA and TKA patients. This is concerning as it represents a significant risk factor for postoperative complication. It additionally confers increased perioperative costs. Patients with OUD should be counseled on their elevated risk, and future work will be needed to determine if this is a modifiable risk factor.
\end{abstract}

\section{Introduction}

The opioid epidemic in the United States is well documented. The United States has experienced an alarming number of drug overdose deaths, and the majority (63\% in 2015) involve opioids [1]. This epidemic, not surprisingly, has garnered attention from the media, government, and medical practitioners. In 2017, the White House declared the opioid crisis a public health emergency. While many of the aforementioned overdoses are a result of illicit drug use, prescription opioid use also contributes significantly [2]. Estimates are that patients treated with opioids for chronic pain misuse their narcotics $21-29 \%$ of the time, and $8-12 \%$ of these patients are addicted to the prescribed drug [3].

In the adult reconstruction realm, prior research has demonstrated that patients prescribed preoperative opioids are at higher risk for perioperative morbidity, revision, and less satisfactory outcomes [4-11]. Much attention has also been paid to postoperative opioid regimens, and there has been increasing recognition that conservative postoperative prescription patterns of opioids can be both safe and effective [12]. However, there has been a substantial increase in the number of patients being prescribed long-term opioids for chronic pain in the community (estimated at a $3-4 \%$ of those treated for 
chronic pain) $[13,14]$. While the majority of these prescriptions are not written by orthopedists [15], surgical candidates taking opioids preoperatively are frequently encountered.

Less common is the patient with a diagnosed opioid use disorder (OUD). This diagnosis is defined by the Diagnostic and Statistical Manual of Mental Disorders 5 (DSM-5) as a problematic pattern of opioid use leading to clinically significant impairment or distress. DSM-IV had previously classified the same disorder as opioid abuse or dependence, and this diagnosis is made when 2 of 11 diagnostic criteria are present in a 12-month period [16]. Much less is known about this patient population, and how frequently these patients are receiving total joint arthroplasty is unknown. Defining risk in these patients is critical, and in value-based reimbursement models, understanding the economic impact of this diagnosis is important.

Therefore, the purpose of this investigation was threefold: (1) we sought to determine the incidence of opioid dependence or abuse in the total hip and knee arthroplasty population, (2) to determine if those with opioid dependence are at higher risk for postoperative complication, and (3) to determine the economic burden associated with this diagnosis. We hypothesized that the incidence of opioid dependence among total joint arthroplasty patients is increasing and that these patients would have higher complication rates and increased resource utilization.

\section{Materials and Methods}

2.1. Data Acquisition. Patients included in this study were selected from the Nationwide Readmission Database (NRD), a publicly available administrative database maintained by the Agency for Healthcare Research and Quality (AHRQ), a part of the Healthcare Cost and Utilization Project (HCUP). Information regarding this database can be found at https:// www.hcup-us.ahrq.gov/tech_assist/centdist.jsp. The NRD includes information on patients of all payer types, including the uninsured. The database provides a nationally representative sample and is able to capture all readmissions occurring in the same state, including readmissions to hospitals different from where the index admission took place. The database contains data on the patient's initial inpatient stay, as well as all subsequent inpatient admissions occurring in the same calendar year. It can thus be queried for postoperative inpatient complication data, as well as diagnoses leading to readmission. This allows for the capture of major complications requiring readmission out to 90 days postoperatively.

Patients were identified using the International Classification of Disease, Ninth Revision, Clinical Modification (ICD-9-CM) procedural codes (total hip arthroplasty 81.51 ; total knee arthroplasty 81.54). The database was queried from 2011 to 2015 quarter 3 (Q3). This was chosen as the end date for this study as this was when the database switched to ICD-10 codes, and exclusion of this transition allowed for equal comparisons. The NRD cannot track patients from year to year, and therefore, we excluded all patients undergoing primary hip or knee arthroplasty in quarter 4 (Q4) of each year as complete 90-day follow-up could not be obtained in these Q4 patients.
ICD-9 diagnosis codes were used to identify patients with opioid use disorders (OUD; abuse or dependence), and the following codes were used: 304.00-304.03, 304.70304.73, and 305.50-305.53. Similarly, postoperative complications were identified using ICD- 9 codes. Hospital costs were calculated using NRD cost-to-charge ratios and were inflation adjusted to the year 2015 using the Consumer Price Index from the Federal Reserve Bank [17].

2.2. Baseline Patient Information. Baseline patient data were collected including the following variables: age, sex, surgery type (THA or TKA), insurance status, zip code income quartile, hospital size, comorbidities, and the presence or not of perioperative transfusion. The included comorbidities were obtained from the Elixhauser Comorbidity Measure [18] and included obesity, diabetes mellitus (DM), preoperative anemia, congestive heart failure (CHF), chronic kidney disease (CKD), chronic obstructive pulmonary disease (COPD), psychiatric illness, and tobacco use. Perioperative transfusion was included in this category as it is well established as a risk factor for postoperative infection [19].

2.3. Outcomes. The incidence of opioid use disorders among total hip and knee arthroplasty patients was calculated on an annual basis by dividing the number of patients with opioid use disorder by the total number of patients undergoing the procedure (with or without OUDs) annually. This was then compared between years. Additionally, we collected information on the following postoperative complications: prosthetic joint infection (PJI), superficial surgical site infection, other wound complications, prosthetic complications (including mechanical complication, loosening, dislocation, prosthetic fracture, and periprosthetic fracture), revision surgery, and postoperative pain (ICD-9 codes 338.18 and 338.19; acute postoperative pain). Complications were included in this study if they occurred on the initial inpatient stay or if they were the cause of readmission. We additionally collected length of stay, readmission, inpatient hospital costs, and 90-day episode costs for all included patients.

2.4. Statistical Analysis. After identification of all patients undergoing THA or TKA, the patients were then divided into two cohorts: patients with and without OUD. HCUPprovided weights were then used to create nationally representative estimates, and further analysis was then conducted. Ultimately, 3,438,105 patients were identified, and of these, $9,832(0.29 \%)$ were found to have a concomitant diagnosis of OUD. First, analysis was conducted comparing baseline patient information between groups using chisquare analysis for categorical values and $t$-tests for continuous variables.

Given the differences identified between nonopioid dependent and OUD patients, statistical matching was performed using coarsened exact matching (CEM) to match patients based on a number of baseline factors. In short, CEM is a validated method of matching [20, 21] that temporarily coarsens data which then allows for exact 
matching. After matching is performed, typical multivariate analysis can then be conducted on matched cohorts. Patients were matched on all of the variables given in Table 1. While many were significantly different prior to matching, after matching was performed, no differences remained $(p \geq 0.997)$. Further analysis was then conducted on these matched cohorts.

Binomial logistic regression, controlling for the variables listed in Table 1, was conducted on matched cohorts to determine if a diagnosis of OUD was an independent predictor of postoperative complications, readmission, or nonhome discharge. Furthermore, cost and LOS were analyzed with linear regression. Additionally, linear regression was used to analyze the trend of incidence of OUD over time. A $p$ value of $<0.05$ was considered significant. All statistics were performed using SPSS Statistics (IBM Corporation, Version 25, Armonk, NY).

\section{Results}

3.1. Baseline Patient Information and the Incidence of Opioid Use Disorder. Ultimately, 3,438,105 (THA: 35\%, and TKA: $65 \%)$ patients were identified for inclusion in this study. 9,982 (0.29\%) patients were identified as having a preexisting OUD (opioid abuse or dependency). Analysis comparing baseline patient information between patients with and without OUD was conducted prior to matching. This analysis revealed that opioid dependent patients included significantly more males, significantly more patients with Medicaid (and less with Medicare or private insurance), significantly younger patients, a higher rate of perioperative transfusion, a higher number of patients in lower income quartile zip codes, and a higher number of patients treated at high-volume centers, as well as significantly different comorbidity profiles $(p<0.001$ for all comparisons) (Table 1).

The incidence of opioid use disorder among those undergoing total hip or knee arthroplasty in the United States was determined by dividing the number of those with OUD each year by the total number of patients undergoing THA or TKA in the corresponding year. We found that the incidence of OUD in arthroplasty patients is increasing significantly, and from 2011 to 2015 , the incidence nearly doubled from $0.2 \%$ to $0.36 \%$ ( $80 \%$ increase, $p<0.001)$ Figure 1.

\subsection{Opioid Use Disorder and Postoperative Complications.} Multivariate analysis conducted on matched cohorts found significant differences in the odds of multiple postoperative complications. These included prosthetic joint infection (OR $1.55,95 \%$ CI 1.23-1.94, $p<0.001$ ), superficial surgical site infection (OR 1.66, 95\% CI 1.34-2.05, $p<0.001$ ), noninfectious wound complication (OR 1.40, 95\% CI 1.12-1.76, $p=0.003$ ), prosthetic complication (OR 1.37, 95\% CI $1.10-1.70, p=0.005$ ), need for early revision surgery (all cause revision; OR 1.47, 95\% CI 1.19-1.81, $p<0.001$ ), and for clinically important acute postoperative pain (OR 2.97, 95\% CI 2.72-3.23, $p<0.001$ ) (Table 2).
3.3. Length of Stay, Nonhome Discharge, 90-Day Readmission, and Economic Burden of Opioid Use Disorder. Multivariate analysis, again controlling for patient characteristics, was conducted to examine postoperative length of stay, nonhome discharge, and 90-day readmission. This analysis revealed that patients with OUD undergoing TKA and THA have significantly longer length of stays (TKA: +0.67 days; THA: +1.09 days, $p<0.001$ ) as well as significantly higher odds of nonhome discharge (TKA: OR 1.47, 95\% CI 1.30-1.64, $p<0.001$; THA: OR $1.40,95 \%$ CI $1.28-1.53, p<0.001$ ) and 90-day readmission (TKA: OR 1.60, 95\% CI 1.43-1.79, $p<0.001$; THA: OR 1.53, 95\% CI $1.43-1.79, p<0.001)$. We additionally compared both inpatient and 90-day costs between patients with and without OUD. This analysis revealed that OUD was associated with significantly increased inpatient (TKA: +\$2,522 [95\% CI $\$ 2,170-2,874]$; THA: $+2,924$ [95\% CI $\$ 2,496-3,353]$, $p<0.001$ ) and total 90-day costs (TKA: $+\$ 3,602[95 \% \mathrm{CI}$ $\$ 3,138-4,065]$; THA: $+4,527$ [95\% CI $\$ 3,593-4,920]$, $p<0.001$ ) in both TKA and THA (Table 3).

\section{Discussion}

The current investigation identifies a clear and concerning increase in the incidence of total hip and knee arthroplasty patients with concomitant opioid use disorders. This is troubling given that our study also demonstrates that this cohort of patients is at elevated perioperative risk for complications, increased length of stay, readmission, and resource utilization.

Our results demonstrated that the incidence of opioid use disorder among total hip and knee arthroplasty patients is increasing on an annual basis. This may be expected given the well-established opioid epidemic [13] along with chronic pain prescribing patterns [14]. A similar analysis conducted on pregnant women demonstrated similar findings with significantly increased rates of opioid use disorders over time [22]. The incidence of OUDs in patients undergoing lumbar fusion was found to be $0.91 \%$, which is higher than our identified rate of $0.36 \%$ in 2015 [23]. This is also lower than the rate that was found in a similar study performed on patients undergoing lower extremity bypass (0.63\%) [24] and lower than the $0.81 \%$ that has been reported for the general population $[24,25]$. The lower rate observed in our cohort likely reflects the fact that patients undergoing total joint arthroplasty are typically older than the general population, and while substance abuse is becoming more common in a geriatric population [26], it remains much more commonly encountered in younger populations. This is also reflected in our two cohorts. Opioid dependent patients were, on average, younger than those without OUD. We controlled for this fact by matching patients on age and subsequently controlling for age in our multivariate regression.

Though novel, our finding that patients with OUD sustain high postoperative morbidity is consistent with prior literature. Similar analyses conducted on patients undergoing lumbar fusion [23] and lower extremity bypass [24] procedures demonstrated similar results, findings that 
TABLe 1: Patient demographics and comorbidities.

\begin{tabular}{|c|c|c|c|c|c|}
\hline \multirow{2}{*}{ Characteristic } & & \multirow{2}{*}{$\begin{array}{l}\text { Nonopioid dependent } \\
(n(\%))\end{array}$} & \multirow{2}{*}{$\begin{array}{l}\text { Opioid dependent } \\
(n(\%))\end{array}$} & \multicolumn{2}{|c|}{$p$ value } \\
\hline & & & & Before matching & After matching \\
\hline Total patients & & $3,428,273(99.7)$ & $9,832(0.3)$ & & \\
\hline \multirow{2}{*}{ Surgery type } & THA & $1,198,470(35)$ & $4,258(43.3)$ & \multirow{2}{*}{$<0.001$} & \multirow{2}{*}{0.999} \\
\hline & TKA & $2,229,803(65)$ & $5,574(56.7)$ & & \\
\hline \multirow{2}{*}{ Sex } & Male & $1,347,632(39.3)$ & $4,693(47.7)$ & \multirow{2}{*}{$<0.001$} & \multirow{2}{*}{0.999} \\
\hline & Female & $2,080,641(60.7)$ & $5,140(52.3)$ & & \\
\hline \multirow{4}{*}{ Insurance } & Medicare & $1,941,276(56.7)$ & $4,576(46.6)$ & \multirow{4}{*}{$<0.001$} & \multirow{4}{*}{0.998} \\
\hline & Private & $1,226,412(35.8)$ & $2,828(28.8)$ & & \\
\hline & Medicaid & $118,405(3.5)$ & $1,869(19.0)$ & & \\
\hline & Other & $137,350(4.0)$ & $552(5.6)$ & & \\
\hline \multirow{5}{*}{ Age category } & $<55$ & $486,664(14.2)$ & $3,550(36.1)$ & \multirow{5}{*}{$<0.001$} & \multirow{5}{*}{0.998} \\
\hline & $55-64$ & $984,362(28.7)$ & $3,852(39.2)$ & & \\
\hline & $65-74$ & $1,191,172(34.7)$ & $1,810(18.4)$ & & \\
\hline & $75-84$ & $653,333(19.1)$ & $540(5.5)$ & & \\
\hline & $85+$ & $112,742(3.3)$ & $81(0.8)$ & & \\
\hline Perioperative transfusion & & $374,279(10.9)$ & $1,463(14.9)$ & $<0.001$ & 0.998 \\
\hline \multirow{4}{*}{ Zip code income quartile } & 1 & $728,949(21.6)$ & $2,348(24.3)$ & \multirow{4}{*}{$<0.001$} & \multirow{4}{*}{0.997} \\
\hline & 2 & $861,933(25.5)$ & $2,602(26.9)$ & & \\
\hline & 3 & $900,568(26.7)$ & $2,523(26.1)$ & & \\
\hline & 4 & $886,595(26.2)$ & $2,189(22.7)$ & & \\
\hline \multirow{3}{*}{ Hospital size } & Low & $741,092(21.6)$ & $1,278(13.0)$ & \multirow{3}{*}{$<0.001$} & \multirow{3}{*}{0.999} \\
\hline & Medium & $866,705(25.3)$ & $2,596(26.4)$ & & \\
\hline & High & $1,820,476(53.1)$ & $5,958(60.6)$ & & \\
\hline \multirow{8}{*}{ Comorbidity } & Obesity & $737,221(21.5)$ & $2,920(29.7)$ & $<0.001$ & 0.999 \\
\hline & Diabetes & $660,785(19.3)$ & $1,626(16.5)$ & $<0.001$ & 0.999 \\
\hline & Psychiatric disorder & $524,428(15.3)$ & $3,961(40.3)$ & $<0.001$ & 0.998 \\
\hline & Anemia & $490,946(14.3)$ & $2,046(20.8)$ & $<0.001$ & 0.999 \\
\hline & CHF & $87,133(2.5)$ & $335(3.4)$ & $<0.001$ & 0.998 \\
\hline & Renal failure & $165,033(4.8)$ & $562(5.7)$ & $<0.001$ & 0.999 \\
\hline & COPD & $507,748(14.8)$ & $2,655(27.0)$ & $<0.001$ & 0.999 \\
\hline & Tobacco use & $268,528(7.8)$ & $3,028(30.8)$ & $<0.001$ & 0.998 \\
\hline
\end{tabular}

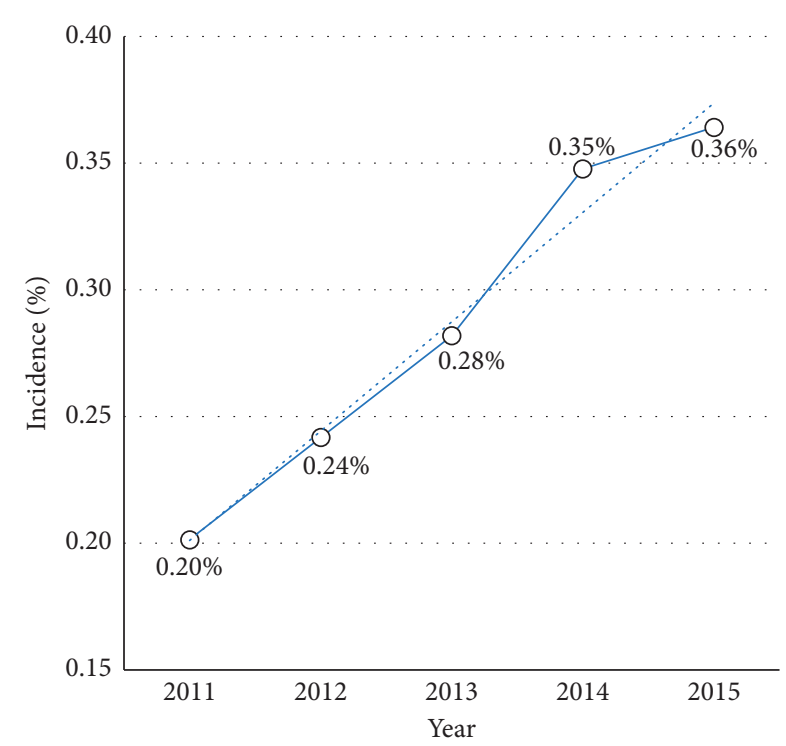

FIGURE 1: The annual incidence of opioid use disorder among patients undergoing total hip or knee arthroplasty in the United States from 2011 to 2015. patients with OUDs had higher rates of complications and nonhome discharge, as well as increased length of stay and total charges. Additionally, patients who are prescribed preoperative opioids before undergoing a THA or TKA are known to be at higher risk for postoperative complications and increased postoperative opioid consumption [4-7, 9-11, 27]. However, until now, analysis of patients with OUD has been limited to small series [28-30]. This population is likely very different than patients simply receiving opioids preoperatively for pain. In fact, the current study found that patients with opioid use disorder who undergo THA or TKA are on average, younger, from lower income zip codes, more likely to be smokers, and have higher comorbid burdens. These are important differences to consider given their impact on postoperative outcomes. While we took multiple steps (statistical matching and subsequently controlling for these differences in our statistical model) to control for these differences in our analysis, it should be recognized that patients with OUD are likely fundamentally different than those without OUD and the entirety of this difference may be difficult to fully account for. However, even when controlling for these differences 
TABLE 2: Multivariate-adjusted analysis of complications in patients with and without opioid use disorder.

\begin{tabular}{lcccc}
\hline Complication & Nonopioid dependent & Opioid dependent & Odds ratio $^{\text {a }}$ & $p$ value (multivariate) \\
\hline Prosthetic joint infection & $22,702(0.7 \%)$ & $190(1.9 \%)$ & $1.55(1.23-1.94)$ & $<0.001$ \\
Superficial surgical site infection & $24,020(0.7 \%)$ & $216(2.2 \%)$ & $1.66(1.34-2.05)$ & $<0.001$ \\
Noninfectious wound complication & $30,492(0.9 \%)$ & $209(2.1 \%)$ & $1.40(1.12-1.76)$ & 0.003 \\
Prosthetic complication & $36,874(1.1 \%)$ & $217(2.2 \%)$ & $1.37(1.10-1.70)$ & 0.005 \\
Revision & $33,965(1.0 \%)$ & $238(2.4 \%)$ & $1.47(1.19-1.81)$ & $<0.001$ \\
Postoperative pain & $135,788(4.0 \%)$ & $1,405(14.3 \%)$ & $2.97(2.72-3.23)$ & $<0.001$ \\
\hline
\end{tabular}

${ }^{a}$ Odds ratio with $95 \%$ confidence interval obtained through multivariate regression on matched cohorts while controlling for patient and operative characteristics.

TABLE 3: Multivariate analysis of healthcare utilization-associated factors.

\begin{tabular}{|c|c|c|c|c|c|}
\hline \multicolumn{2}{|c|}{ Complication } & Nonopioid dependent $\mathrm{t}^{\mathrm{a}}$ & Opioid dependent ${ }^{\mathrm{a}}$ & Beta coefficient or odds ratio ${ }^{b}$ & $p$ value (multivariate) \\
\hline \multirow{5}{*}{ TKA } & Nonhome discharge & $621,157(27.9 \%)$ & $1,729(31.0 \%)$ & $1.47(1.30-1.64)$ & $<0.001$ \\
\hline & 90-day readmission & $190,769(8.6 \%)$ & $823(14.8 \%)$ & $1.60(1.43-1.79)$ & $<0.001$ \\
\hline & Length of stay (days) & $3.43 \pm 2.74$ & $4.39 \pm 4.22$ & +0.67 days $(0.56-0.78)$ & $<0.001$ \\
\hline & Inpatient cost (USD) & $\$ 17,013 \pm 8,923$ & $\$ 20,394 \pm 11,067$ & $+\$ 2,522(2,170-2,874)$ & $<0.001$ \\
\hline & Total 90-day cost (USD) & $\$ 18,250 \pm 11,575$ & $\$ 22,873 \pm 15,793$ & $+\$ 3,602(3,138-4,065)$ & $<0.001$ \\
\hline \multirow{5}{*}{ THA } & Nonhome discharge & $327,408(27.3 \%)$ & $1,357(31.9 \%)$ & $1.40(1.28-1.53)$ & $<0.001$ \\
\hline & 90-day readmission & $112,624(9.4 \%)$ & $777(18.2 \%)$ & $1.53(1.43-1.79)$ & $<0.001$ \\
\hline & Length of stay (days) & $3.48 \pm 3.59$ & $5.26 \pm 9.40$ & +1.09 days $(0.95-1.24)$ & $<0.001$ \\
\hline & Inpatient cost (USD) & $\$ 17,943 \pm 9,799$ & $\$ 22,307 \pm 15,385$ & $+\$ 2,924(2,496-3,353)$ & $<0.001$ \\
\hline & Total 90-day cost (USD) & $\$ 19,603 \pm 13,252$ & $\$ 26,198 \pm 21,360$ & $+\$ 4,257(3,593-4,920)$ & $<0.001$ \\
\hline
\end{tabular}

${ }^{a}$ Presented as mean \pm standard deviation for continuous variables or $n$ (\%) for categorical variables, and univariate values represent unmatched cohorts. ${ }^{b}$ Odds ratio or beta coefficient with $95 \%$ confidence interval obtained through multivariate regression on matched cohorts while controlling for patient and operative characteristics; beta represents an increase in days and dollars associated with an opioid use disorder.

and performing analysis on matched cohorts, rates of complication were significantly higher in the OUD cohort.

Infectious complications have previously been associated with substance abuse, as Bauer et al. found nearly a $40 \%$ infection rate in patients who illicitly use intravenous drugs after TKA [31]. Preoperative opioid use has been repeatedly associated with surgical site infections in arthroplasty $[9,11,27]$ as well as other general surgery procedures $[32,33]$. Why this may be the case is likely multifactorial and could include patient and socioeconomic factors. Nonetheless, some limited evidence exists to suggest that opioids are associated with delayed wound healing which could be partially responsible for some of our observed associations [34]. This is further supported by the finding that OUD patients had significantly higher rates of wound healing issues-both infectious and noninfectious. Direct impairment of immune cells by opioids has also been demonstrated in vitro and in animal models [35].

A significant finding of this investigation is that OUD represents an expensive comorbidity. As value-based models emerge, this is an important consideration and adjustments will need to be made for patients with OUD as they are at increased risk for readmission, complications, and subsequent increased costs. In this study, patients with OUD were significantly more costly. This was by over $\$ 2500+$ for inpatient costs and $\$ 3600+$ for total 90 -day costs in both THA and TKA. These differences persisted, even when controlling for demographic, procedural, and comorbid data. Alternatively, we do not suggest that prior OUD is a contraindication to arthroplasty as prior studies demonstrate these patients do improve [6]. However, these patients must be recognized and counseled that they are at high risk, and when possible, should be weaned from narcotics preoperatively to reduce their risk of complications $[9,29]$.

There are multiple limitations to this investigation, most of which are inherent to analysis of large administrative databases. First, our analysis is reliant on accurate coding. It is possible that some patients with opioid abuse or dependence were not coded as such and were therefore included in the incorrect cohort. The same is true of the preoperative comorbidities included in our match and as controls in our multivariate model as well as our examined outcomes. On the same note, we assumed in our analysis that coding frequency stayed the same over time. Our observed increase in incidence, therefore, could be partially accounted for by an increased awareness of the opioid epidemic and increased coding for OUD over time. Similarly, the NRD reports only on patients who were treated as inpatients. Given the movement to outpatient total joint arthroplasty, this likely means that many patients were not captured. However, inclusion of patients treated as outpatients may have actually increased our observed differences between cohorts as these are generally healthy patients with low-risk profiles. Generally, opioid use disorder would be considered a contraindication to outpatient arthroplasty [36] and it, therefore, is likely that the vast majority of these patients have been captured in this investigation. Additionally, using the NRD, only inpatient complications are captured unless the complication leads to readmission and even readmissions are tracked only to 90 days. This could mean that our complication rates are underestimated. Last, it is true that complete isolation of OUD may be difficult. Still, 
this analysis was performed on matched cohorts and controlling for available comorbid, socioeconomic, and procedural data, likely providing the best analysis possible.

Despite its limitations, the use of the Nationwide Readmission Database is a strength of this study as it allows for national estimates and provides a very large, all-payer sample. It additionally allows for controlling of socioeconomic factors by reporting insurance status as well as zip code income quartile. Both factors that have been associated with outcomes [37-39], and the ability to control for these represents an important strength compared to other database studies. Also, readmissions can be tracked even if the readmission occurs at a different institution, therefore providing a higher capture rate for outcomes. Additionally, trends in incidence of OUD in the arthroplasty population could not confidently be reported without the use of a large administrative database, representing an advantage over single-institution studies. Finally, the NRD provides chargecost ratios and reports economic data allowing for quantification of the additional cost conferred by OUD as a comorbid condition. As value-based reimbursement models emerge and evolve, this information is invaluable.

In conclusion, opioid use disorder as a preoperative comorbid condition is becoming increasingly common in the primary total hip and total knee arthroplasty patients. This is an important finding as this patient cohort has elevated risk for multiple important complications, readmission, extended length of stay, and increased economic burden in the early postoperative period. This patient population must be counseled on their elevated risks. Furthermore, this study adds to the evidence suggesting these patients should be weaned from preoperative narcotics, as opioid freedom prior to surgery, even after longterm use, confers a risk reduction $[6,7,9,27]$. Future research may be directed at evaluating preoperative opioid use in morphine equivalents to determine cutoff values for safe surgery.

\section{Data Availability}

The data used for this study are publicly available for a fee at the following web address: https://www.hcup-us.ahrq.gov/ tech_assist/centdist.jsp.

\section{Conflicts of Interest}

The authors declare that they have no conflicts of interest.

\section{References}

[1] P. Seth, L. Scholl, R. A. Rudd, and S. Bacon, "Overdose deaths involving opioids, cocaine, and psychostimulants-United States, 2015-2016," MMWR. Morbidity and Mortality Weekly Report, vol. 67, no. 12, pp. 349-358, 2018.

[2] A. D. Kaye, M. R. Jones, A. M. Kaye et al., "Prescription opioid abuse in chronic pain: an updated review of opioid abuse predictors and strategies to curb opioid abuse: Part 1," Pain Physician, vol. 20, no. 2S, pp. S93-S109, 2017.

[3] K. E. Vowles, M. L. McEntee, P. S. Julnes, T. Frohe, J. P. Ney, and D. N. van der Goes, "Rates of opioid misuse, abuse, and addiction in chronic pain," Pain, vol. 156, no. 4, pp. 569-576, 2015.

[4] J. C. Rozell, P. M. Courtney, J. R. Dattilo, C. H. Wu, and G.-C. Lee, "Preoperative opiate use independently predicts narcotic consumption and complications after total joint arthroplasty," The Journal of Arthroplasty, vol. 32, no. 9, pp. 2658-2662, 2017.

[5] S. R. Smith, J. Bido, J. E. Collins, H. Yang, J. N. Katz, and E. Losina, "Impact of preoperative opioid use on total knee arthroplasty outcomes," The Journal of Bone and Joint Surgery, vol. 99, no. 10, pp. 803-808, 2017.

[6] C. M. Goplen, W. Verbeek, S. H. Kang et al., "Preoperative opioid use is associated with worse patient outcomes after total joint arthroplasty: a systematic review and meta-analysis," BMC Musculoskelet Disord, vol. 20, no. 1, p. 234, 2019.

[7] A. Ben-Ari, H. Chansky, and I. Rozet, "Preoperative opioid use is associated with early revision after total knee arthroplasty," The Journal of Bone and Joint Surgery, vol. 99, no. 1, pp. 1-9, 2017.

[8] J. Starr, I. Rozet, and A. Ben-Ari, "A risk calculator using preoperative opioids for prediction of total knee revision arthroplasty," The Clinical Journal of Pain, vol. 34, no. 4, pp. 328-331, 2018.

[9] N. Jain, J. L. Brock, A. T. Malik, F. M. Phillips, and S. N. Khan, "Prediction of complications, readmission, and revision surgery based on duration of preoperative opioid use," The Journal of Bone and Joint Surgery, vol. 101, no. 5, pp. 384-391, 2019.

[10] J. Weick, H. Bawa, D. R. Dirschl, and H. H. Luu, "Preoperative opioid use is associated with higher readmission and revision rates in total knee and total hip arthroplasty," The Journal of Bone and Joint Surgery, vol. 100, no. 14, pp. 1171-1176, 2018.

[11] K. L. Bell, N. Shohat, K. Goswami, T. L. Tan, I. Kalbian, and J. Parvizi, "Preoperative opioids increase the risk of periprosthetic joint infection after total joint arthroplasty," The Journal of Arthroplasty, vol. 33, no. 10, pp. 3246-3251.e1, 2018.

[12] A. J. Holte, C. N. Carender, N. O. Noiseux, J. E. Otero, and T. S. Brown, "Restrictive opioid prescribing protocols following total hip arthroplasty and total knee arthroplasty are safe and effective," The Journal of Arthroplasty, vol. 34, no. 7, pp. S135-S139, 2019.

[13] N. D. Volkow and A. T. McLellan, "Opioid abuse in chronic pain-misconceptions and mitigation strategies," New England Journal of Medicine, vol. 374, no. 13, pp. 1253-1263, 2016.

[14] D. Boudreau, M. Von Korff, C. M. Rutter et al., "Trends in long-term opioid therapy for chronic non-cancer pain," Pharmacoepidemiology and Drug Safety, vol. 18, no. 12, pp. 1166-1175, 2009.

[15] R. S. Namba, E. W. Paxton, and M. C. Inacio, "Opioid prescribers to total joint arthroplasty patients before and after surgery: the majority are not orthopedists," The Journal of Arthroplasty, vol. 33, no. 10, pp. 3118-3124.e3, 2018.

[16] American Psychiatric Association, Diagnostic and Statistical Manual of Mental Disoders: DSM-5, American Psychiatric Association Publishing, Washington, DC, USA, 2013.

[17] S. L. Fed, "Economic research: consumer price indexes (CPI and PCE)," https://fred.stlouisfed.org/categories/9.

[18] A. Elixhauser, C. Steiner, D. R. Harris, and R. M. Coffey, "Comorbidity measures for use with administrative data," Medical Care, vol. 36, no. 1, pp. 8-27, 1998.

[19] J. L. Kim, J.-H. Park, S.-B. Han, I. Y. Cho, and K.-M. Jang, "Allogeneic blood transfusion is a significant risk factor for 
surgical-site infection following total hip and knee arthroplasty: a meta-analysis," The Journal of Arthroplasty, vol. 32, no. 1, pp. 320-325, 2017.

[20] S. M. Iacus, G. King, and G. Porro, "Causal inference without balance checking: coarsened exact matching," Political Analysis, vol. 20, no. 1, pp. 1-24, 2012.

[21] S. M. Iacus, G. King, and G. Porro, "Multivariate matching methods that are monotonic imbalance bounding," Journal of the American Statistical Association, vol. 106, no. 493, pp. 345-361, 2011.

[22] S. C. Haight, J. Y. Ko, V. T. Tong, M. K. Bohm, and W. M. Callaghan, "Opioid use disorder documented at delivery hospitalization-United States, 1999-2014," MMWR. Morbidity and Mortality Weekly Report, vol. 67, no. 31, pp. 845-849, 2018.

[23] M. L. Martini, D. A. Nistal, B. C. Deutsch, and J. M. Caridi, "Characterizing the risk and outcome profiles of lumbar fusion procedures in patients with opioid use disorders: a step toward improving enhanced recovery protocols for a unique patient population," Neurosurgical Focus, vol. 46, no. 4, p. E12, 2019.

[24] M. Aizpuru, L. K. Gallo, K. X. Farley et al., "Economic burden and clinical impact of pre-operative opioid dependence for patients undergoing lower extremity bypass surgery," Journal of Vascular Surgery, 2019, in Press.

[25] Medicine ASoA, "Opioid addiction: 2016 facts and figures," 2016, https://www.asam.org/docs/default-source/advocacy/ opioid-addiction-disease-facts-figures.pdf.

[26] S. Yarnell, L. Li, B. MacGrory, L. Trevisan, and P. Kirwin, "Substance use disorders in later life: a review and synthesis of the literature of an emerging public health concern," The American Journal of Geriatric Psychiatry, 2019, in Press.

[27] M. Blevins Peratikos, H. L. Weeks, A. J. B. Pisansky, R. J. Yong, and E. A. Stringer, "Effect of preoperative opioid use on adverse outcomes, medical spending, and persistent opioid use following elective total joint arthroplasty in the United States: a large retrospective cohort study of administrative claims data," Pain Medicine, 2019.

[28] C. R. Lehman, M. D. Ries, G. D. Paiement, and A. B. Davidson, "Infection after total joint arthroplasty in patients with human immunodeficiency virus or intravenous drug use," The Journal of Arthroplasty, vol. 16, no. 3, pp. 330-335, 2001.

[29] L.-C. L. Nguyen, D. C. Sing, and K. J. Bozic, "Preoperative reduction of opioid use before total joint arthroplasty," The Journal of Arthroplasty, vol. 31, no. 9, pp. 282-287, 2016.

[30] K. Wieser, P. O. Zingg, M. Betz, G. Neubauer, and C. Dora, "Total hip replacement in patients with history of illicit injecting drug use," Archives of Orthopaedic and Trauma Surgery, vol. 132, no. 7, pp. 1037-1044, 2012.

[31] D. E. Bauer, A. Hingsammer, L. Ernstbrunner et al., "Total knee arthroplasty in patients with a history of illicit intravenous drug abuse," International Orthopaedics, vol. 42, no. 1, pp. 101-107, 2018.

[32] D. C. Cron, M. J. Englesbe, C. J. Bolton et al., "Preoperative opioid use is independently associated with increased costs and worse outcomes after major abdominal surgery," Annals of Surgery, vol. 265, no. 4, pp. 695-701, 2017.

[33] A. D. Harris, B. Fleming, J. S. Bromberg et al., "Surgical site infection after renal transplantation," Infection Control \& Hospital Epidemiology, vol. 36, no. 4, pp. 417-423, 2015.

[34] V. K. Shanmugam, K. S. Couch, S. McNish, and R. L. Amdur, "Relationship between opioid treatment and rate of healing in chronic wounds," Wound Repair and Regeneration, vol. 25, no. 1, pp. 120-130, 2017.

[35] L. M. Plein and H. L. Rittner, "Opioids and the immune system-friend or foe," British Journal of Pharmacology, vol. 175 , no. 14 , pp. 2717-2725, 2018.

[36] R. M. Meneghini, M. Ziemba-Davis, M. K. Ishmael, A. L. Kuzma, and P. Caccavallo, "Safe selection of outpatient joint arthroplasty patients with medical risk stratification: the "outpatient arthroplasty risk assessment score"," The Journal of Arthroplasty, vol. 32, no. 8, pp. 2325-2331, 2017.

[37] D. Shau, N. Shenvi, K. Easley, M. Smith, T. Bradbury, and G. Guild III, "Medicaid payer status is associated with increased 90-day morbidity and resource utilization following primary total hip arthroplasty," The Journal of Bone and Joint Surgery, vol. 100, no. 23, pp. 2041-2049, 2018.

[38] D. Shau, N. Shenvi, K. Easley, M. Smith, and G. Guild III, "Medicaid is associated with increased readmission and resource utilization after primary total knee arthroplasty: a propensity score-matched analysis," Arthroplasty Today, vol. 4, no. 3, pp. 354-358, 2018.

[39] P. M. Courtney, J. I. Huddleston, R. Iorio, and D. C. Markel, "Socioeconomic risk adjustment models for reimbursement are necessary in primary total joint arthroplasty," The Journal of Arthroplasty, vol. 32, no. 1, pp. 1-5, 2017. 


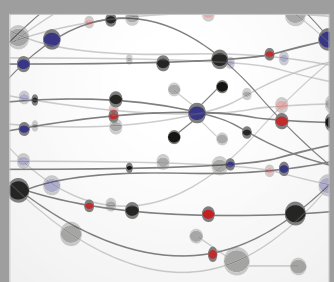

The Scientific World Journal
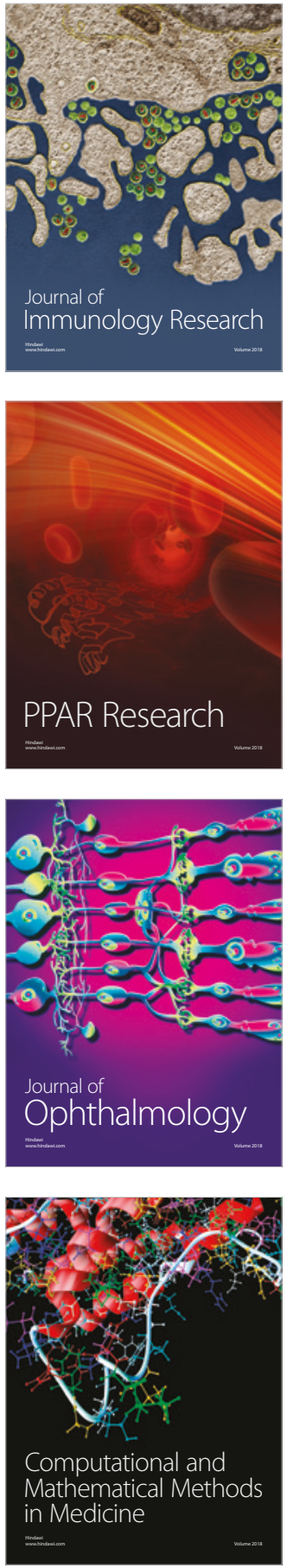

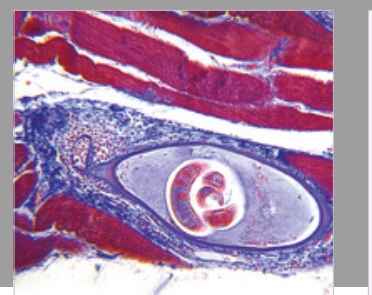

Gastroenterology Research and Practice

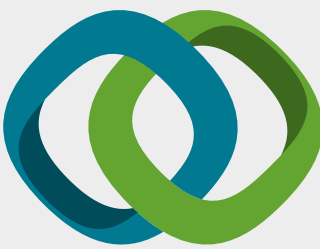

\section{Hindawi}

Submit your manuscripts at

www.hindawi.com
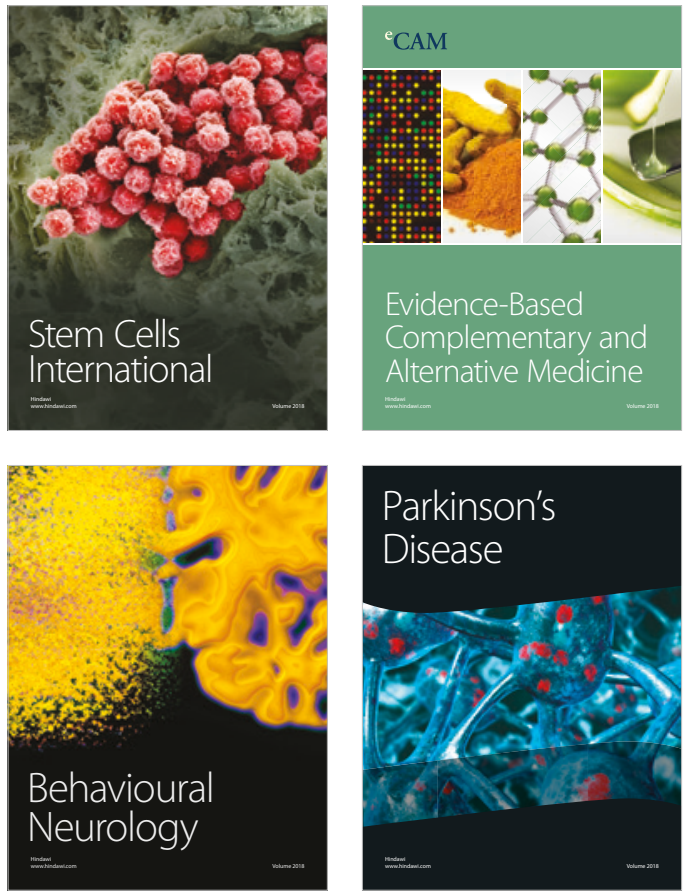

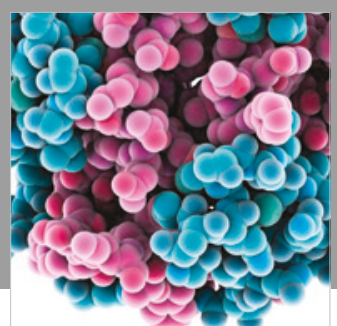

ournal of

Diabetes Research

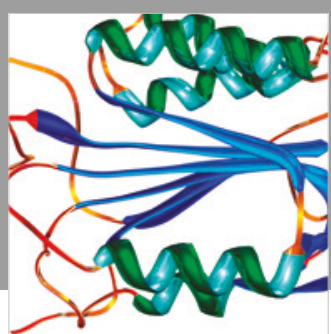

Disease Markers
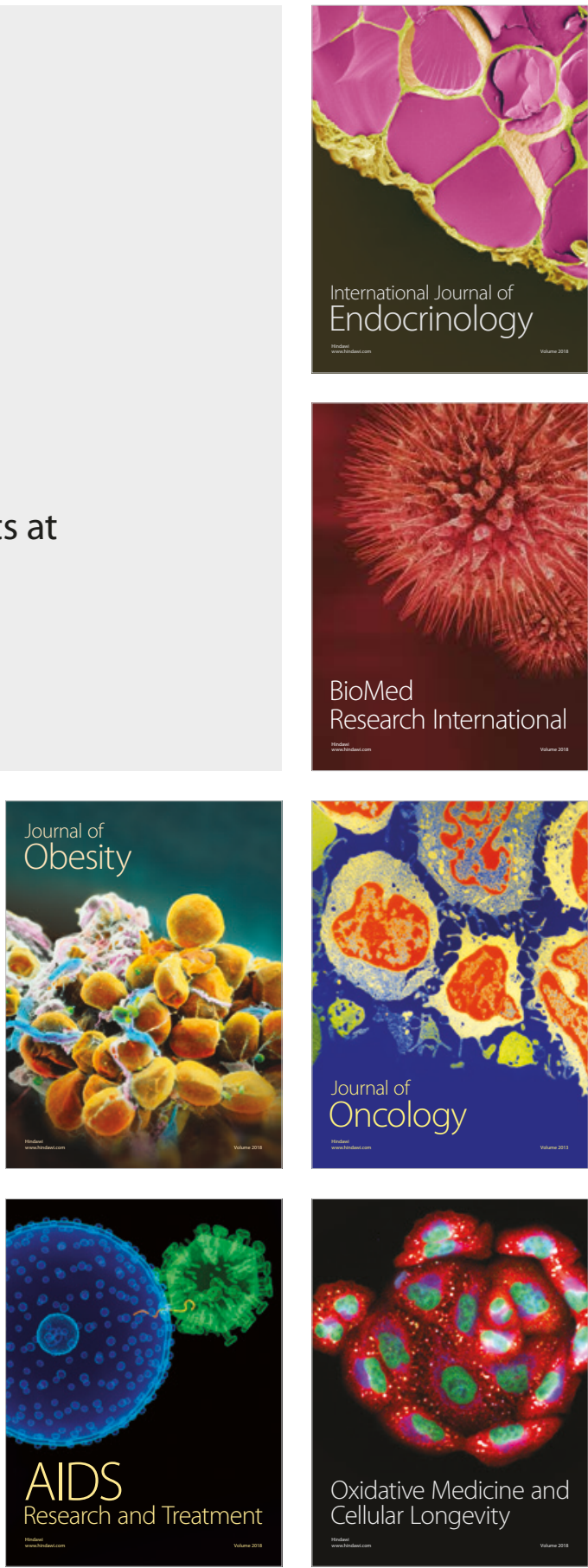